\title{
Molecularly targeted therapies for asthma: current development, challenges and potential clinical translation
}

\begin{abstract}
Extensive research into the therapeutics of asthma has yielded numerous effective interventions over the past few decades. However, adverse effects and ineffectiveness of most of these medications especially in the management of steroid resistant severe asthma necessitate the development of better medications. Numerous drug targets with inherent airway smooth muscle tone modulatory role have been identified for asthma therapy. This article reviews the latest understanding of underlying molecular aetiology of asthma towards design and development of better antiasthma drugs. New drug candidates with their putative targets that have shown promising results in the preclinical and/or clinical trials are summarised. Examples of these interventions include restoration of $\mathrm{Th}_{1} / \mathrm{Th}_{2}$ balance by the use of newly developed immunomodulators such as toll-like receptor-9 activators (CYT003QbG10 and QAX-935). Clinical trials revealed the safety and effectiveness of chemoattractant receptor-homologous molecule expressed on Th2 cells (CRTH2) antagonists such as OC0000459, BI-671800 and ARRY-502 in the restoration of $\mathrm{Th}_{1} / \mathrm{Th}_{2}$ balance. Regulation of cytokine activity by the use of newly developed biologics such as benralizumab, reslizumab, mepolizumab, lebrikizumab, tralokinumab, dupilumab and brodalumab are at the stage of clinical development. Transcription factors are potential targets for asthma therapy, for example SB010, a GATA-3 DNAzyme is at its early stage of clinical trial. Other candidates such as inhibitors of Rho kinases (Fasudil and Y-27632), phosphodiesterase inhibitors (GSK256066, CHF 6001, roflumilast, RPL 554) and proteinase of activated receptor-2 (ENMD-1068) are also discussed. Preclinical results of blockade of calcium sensing receptor by the use of calcilytics such as calcitriol abrogates cardinal signs of asthma. Nevertheless, successful translation of promising preclinical data into clinically viable interventions remains a major challenge to the development of novel anti-asthmatics.
\end{abstract}

Keyword: Asthma; Inflammation; Airway remodeling; Anti-asthmatics; Targets 